
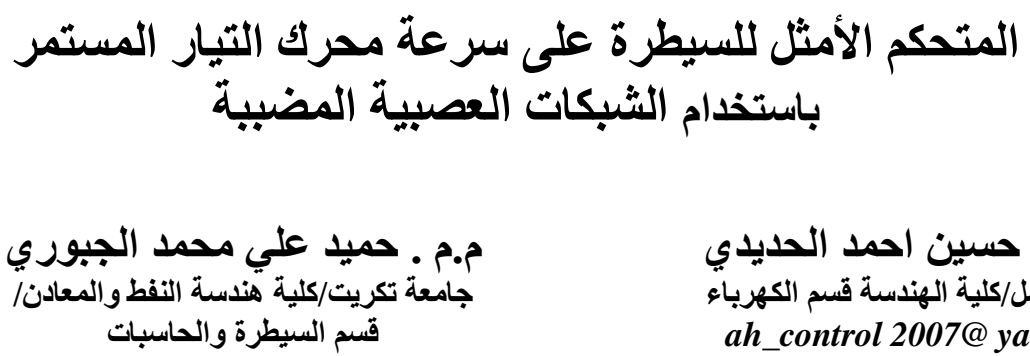

hamed.ali8822@yahoo.com:

\title{
الخلاصة
}

العمل الحالي يتتاول دراسة سرعة محرك تيار مستمر مسيطر عليه باستخدام المغير الخافض الرافع واختبار نظام الحلقة

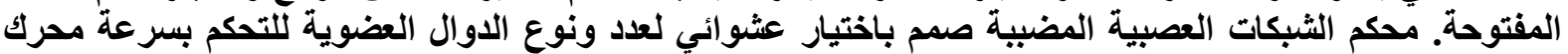

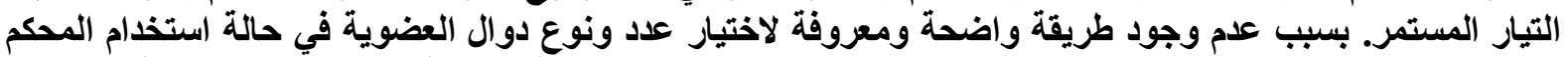

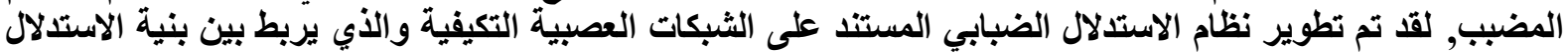

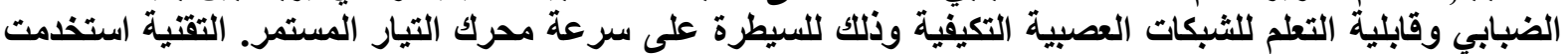

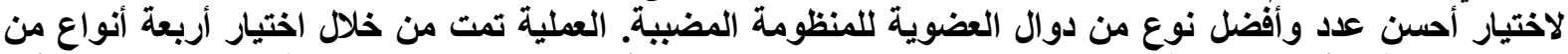

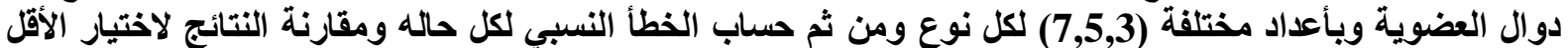

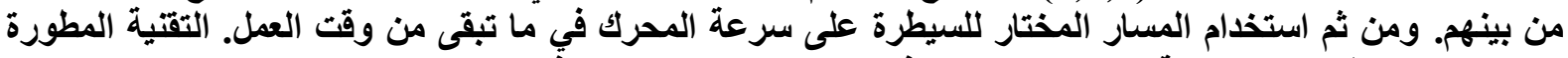

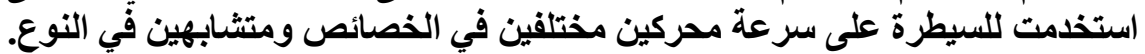
الكلمات الدالة:الثبكات العصبية المضبية , محرك التيار المستمر , السيطرة التكيفية , المغير الخافض التئين الرافع.

\section{Optimal Neuro-Fuzzy D.C. Motor Speed Control}

\section{Assist proof. Dr. Ali H. Al-}

Elect. Eng. Dept. Collage of Eng. University of Mosul

\section{Assist lecture. Hameed A.}

Control \& Computer Eng. Dept. College of Petroleum \& Minerals Eng. Tikrit University

\begin{abstract}
This work deals with investigating of d.c. motor speed controlled by a buck-boost converter. Open loop system is tested. Aneuro-Fuzzy controller with random number and type of membership function is designed to control the speed of the d.c. motor as a closed loop system. Because of the lack of a clear and a known way for selecting the type and number of membership function in case of fuzzy control, An Adaptive NeuroFuzzy Inference System which comprises a fuzzy inference structure and neural network learning ability is modified to control the speed of the motor. The technique is used to select the optimal number and the best type of membership function for the fuzzy system. The process is carried out through testing four types of membership functions with different numbers $(3,5, \ldots$ etc) for each type and computing the absolute error for each case comparing their results to choose the smallest among them. Then the chosen root is applied to control the system for the rest time of control. The technique is applied to two loads (motors).
\end{abstract}


تلعب المحركات الكهربائية دور اً مهماً في الصناعة, حيث تستعمل لتوليد في السيار ات الكهربائية في محطات القدرة

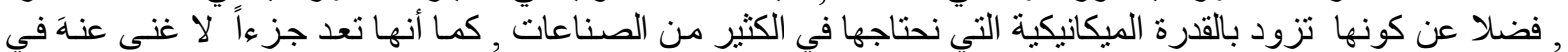

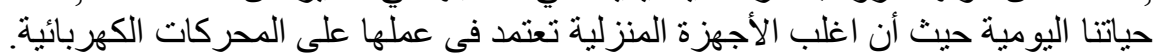

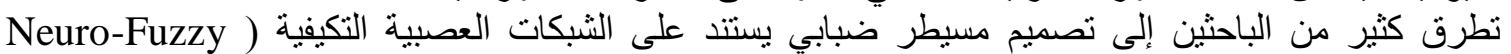
(Controller

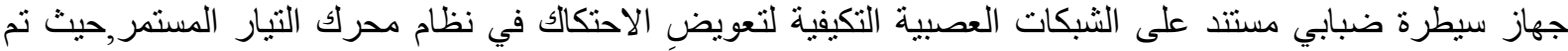
اختيار خمس دو ال عضوية نوع (Gaussian) وبشكل عشو ائي. كما قام الباحثون (Adriana Florescu, Ovidiu ) (Grigore, Dan Alexandru Stoichescu, Alexandru Vasile

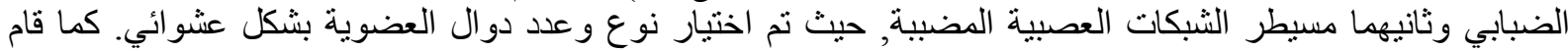
الباحثون (Ferenc Farkas, Sandor Halasz, Istvan Kadar) بعرض مسيطر الثبكات العصبية المضبية للسيطرة

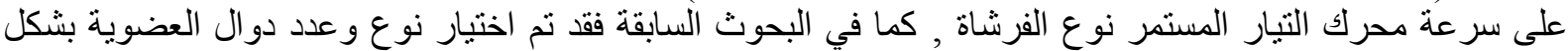

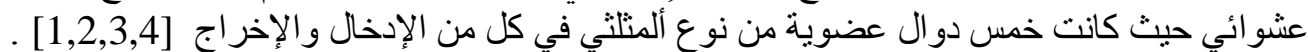

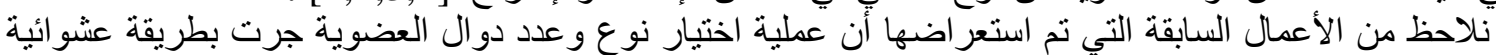

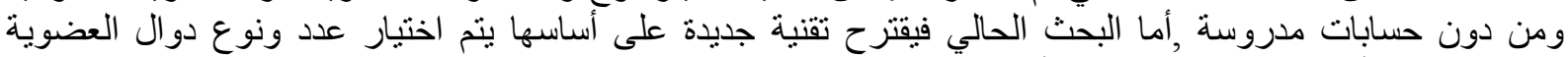

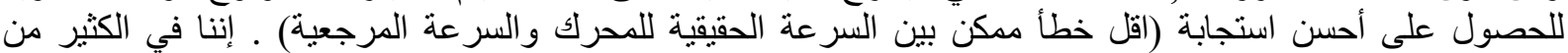

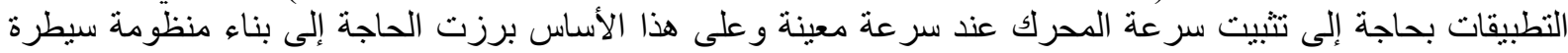

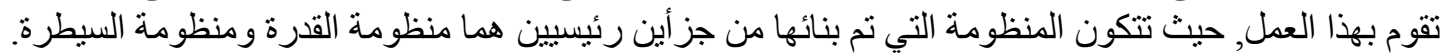

Power System 1

تتكون منظومة ألقدرة من محرك التمرة التيار المستمر, ومغير التيار المستمر الخافض الر افع (Buck Boost Converter):

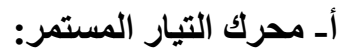
في هذا البحث تم الئيار المتيار نموذجين من محرك التيار المستمر ذي الإثارة المنفصلة, حيث الثكل (1) يبين الدائرة الكهربائية المكافئة لكل من المنتج و المجال للمحرك.

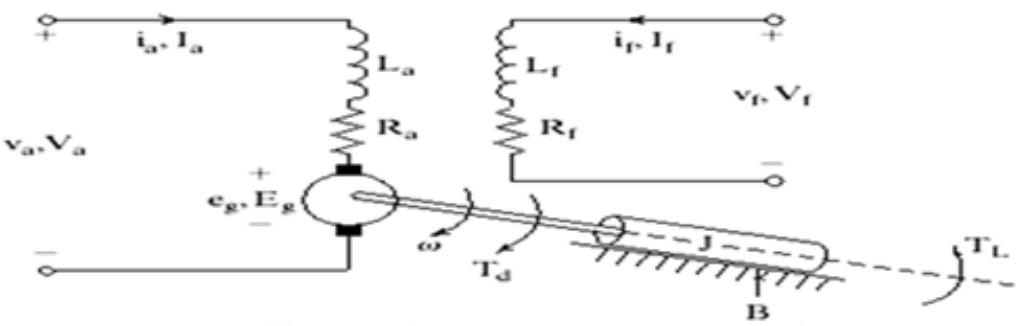

شكل(1) الدائرة الكهربائية المكافئة لمحرك تيار مستمر منفصل الإثارة

وان المصفوفات التي تصف عمل المحرك فهي كما مبين في أدناه:

$\left[\begin{array}{l}\dot{i}_{a} \\ \dot{\omega}_{m}\end{array}\right]=\left[\begin{array}{cc}\frac{-R_{a}}{L_{a}} & \frac{-k_{b}}{L_{a}} \\ \frac{k_{b}}{J} & \frac{-B}{J}\end{array}\right]\left[\begin{array}{l}i_{a} \\ w_{m}\end{array}\right]+\left[\begin{array}{cc}\frac{1}{L_{a}} & 0 \\ 0 & \frac{-1}{J}\end{array}\right]\left[\begin{array}{c}v_{a} \\ T_{L}\end{array}\right]$

i ia

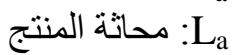

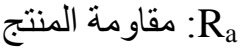

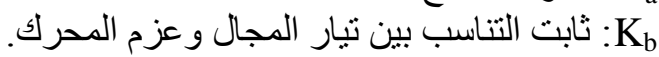

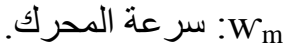

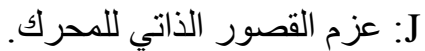




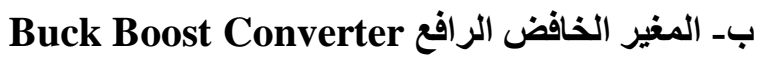

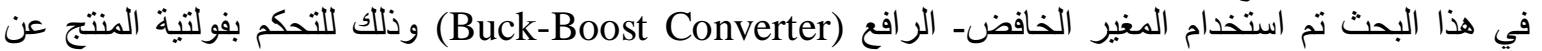

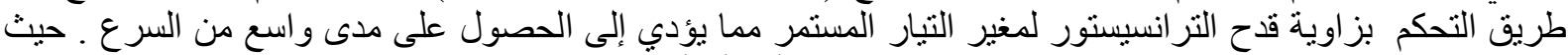

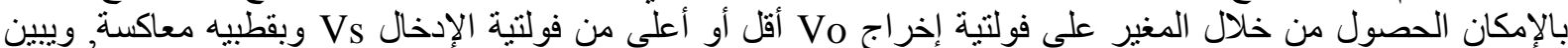

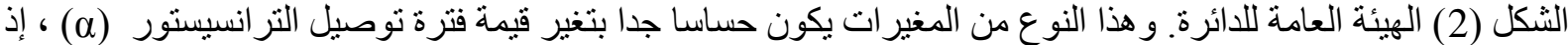

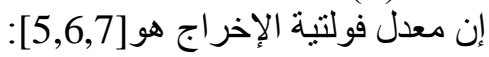

$V o=-\frac{\alpha \cdot V s}{(1-\alpha)}$

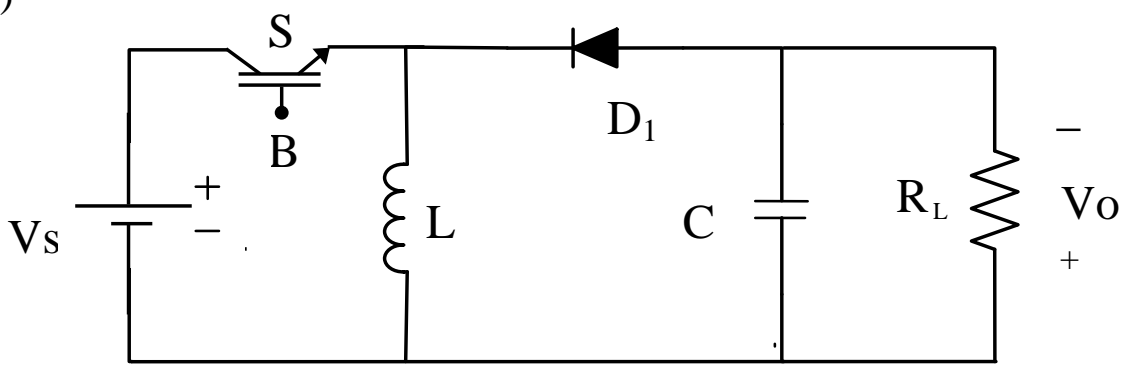

الثكل(2) الهيئة العامة لدائرة المغير الخافض الرافع

بالإمكان كتابة مصفوفات عامة تضم حالتي عمل التر انسيستور وبدلالة نسبة فترة التوصيل( م) كما مبين أدناه [5]. $\left[\begin{array}{c}\dot{i}_{L} \\ \dot{v}_{C}\end{array}\right]=\left[\begin{array}{cc}0 & \frac{-(1-d)}{L} \\ \frac{(1-d)}{C} & \frac{-1}{\left(R_{L} \cdot C\right)}\end{array}\right]\left[\begin{array}{l}i_{L} \\ v_{C}\end{array}\right]+\left[\begin{array}{l}\frac{d}{L} \\ 0\end{array}\right] \cdot V_{S}$

جـــــالمعادلات الرياضية العامة للنظام:

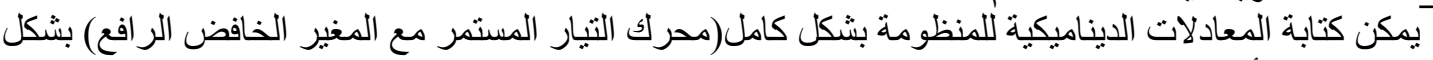
مصفوفات و كما يأتي: $\left[\begin{array}{l}x_{1} \\ x_{2} \\ x_{3} \\ x_{4}\end{array}\right]=\left[\begin{array}{cccc}0 & -\frac{(1-\alpha)}{L} & 0 & 0 \\ \frac{(1-\alpha)}{C} & \frac{-1}{\left(R_{L} \cdot C\right)} & 0 & 0 \\ 0 & \frac{1}{L_{a}} & \frac{-R_{a}}{L_{a}} & \frac{-k_{b}}{L_{a}} \\ 0 & 0 & \frac{k_{b}}{J} & \frac{-B}{J}\end{array}\right]\left[\begin{array}{c}x_{1} \\ x_{2} \\ x_{3} \\ x_{4}\end{array}\right]+\left[\begin{array}{cc}\frac{\alpha}{L} & 0 \\ 0 & 0 \\ 0 & 0 \\ 0 & \frac{-1}{J}\end{array}\right]\left[\begin{array}{c}V_{S} \\ T_{L}\end{array}\right] y=\left[\begin{array}{llll}0 & 0 & 0 & 1\end{array}\right]\left[\begin{array}{l}x_{1} \\ x_{2} \\ x_{3} \\ x_{4}\end{array}\right]$ دـ استجابة نظام الدائرة المفتوحة:

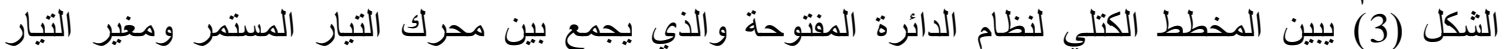

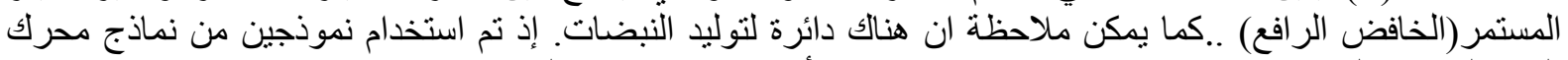

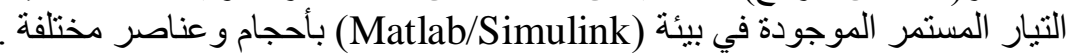




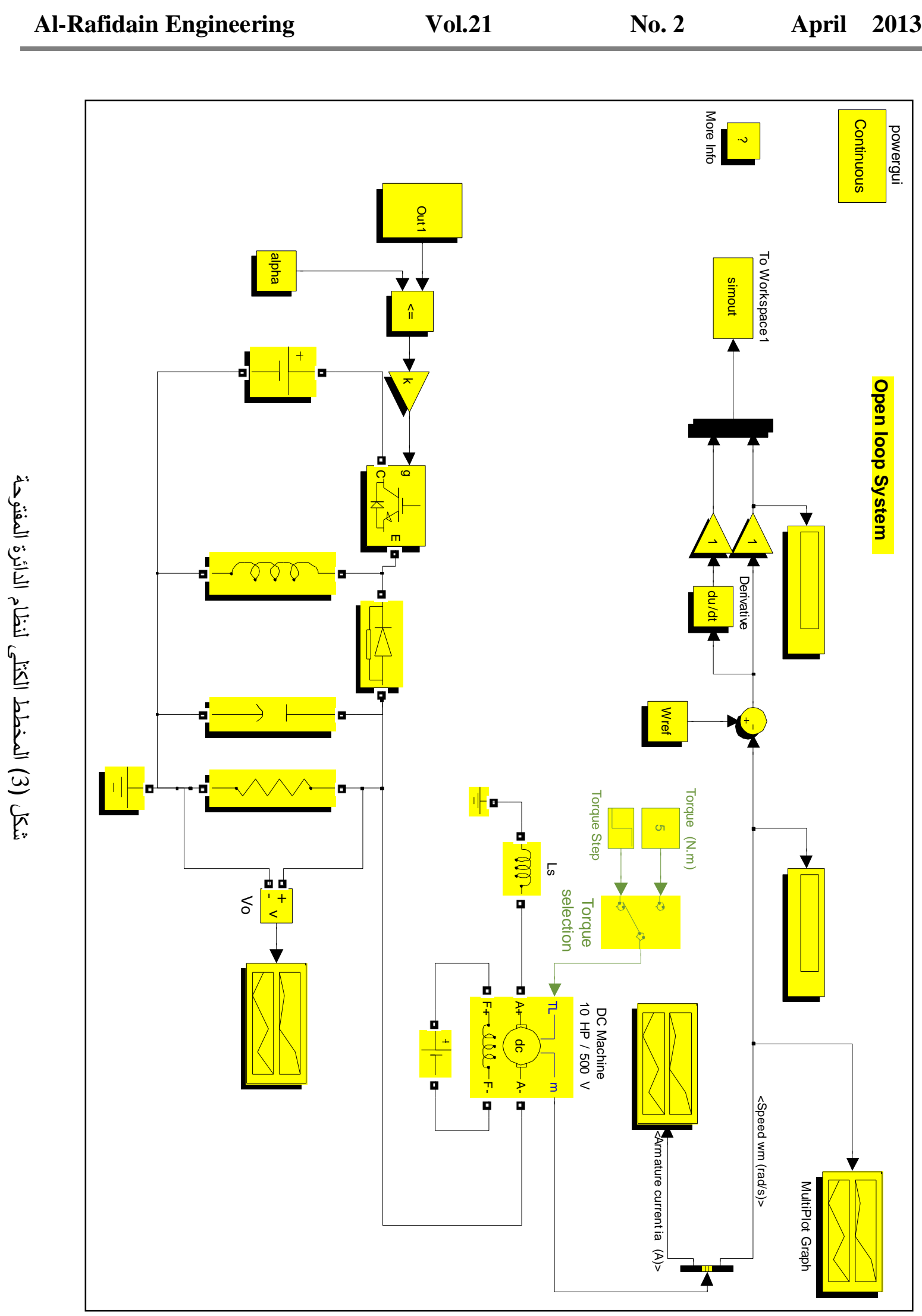


الشكل (4) يبين استجابة محرك التيار المستمر كنظام دائرة مفتوحة , فلقد تم تسليط نسبة فترة توصيل بمقدار

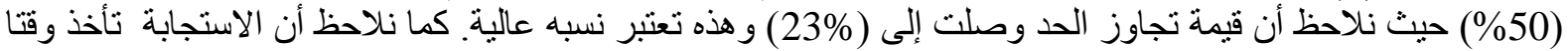

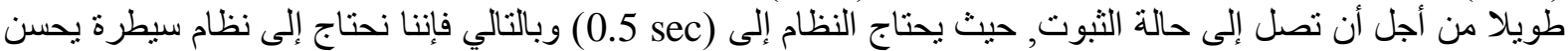

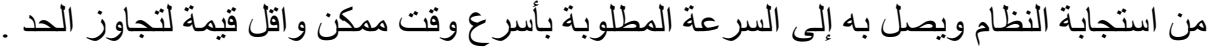

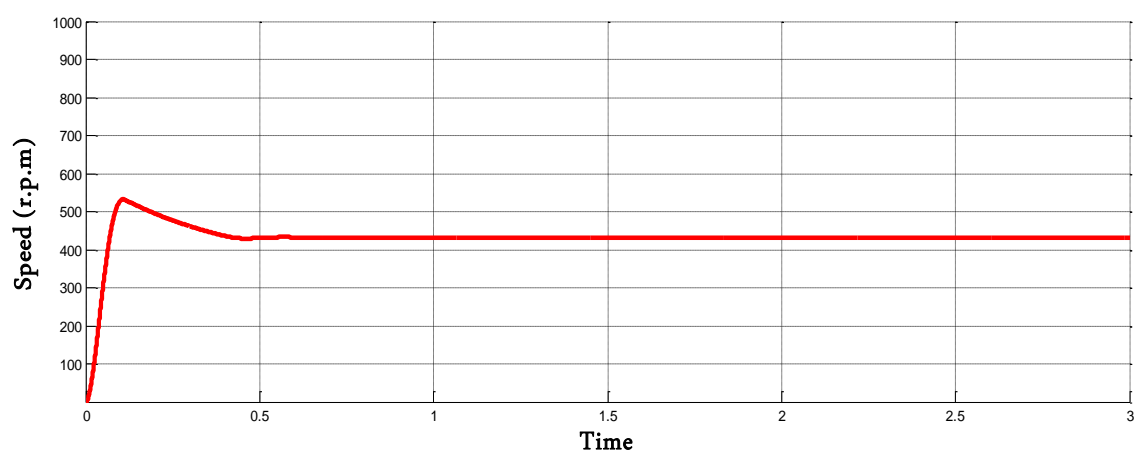

$$
\text { شكل(4) استجابة سرعة المحرك الاول عند (a=50\%) }
$$

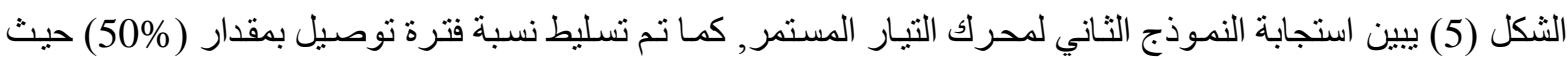

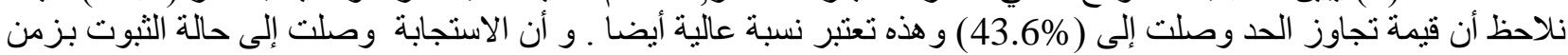

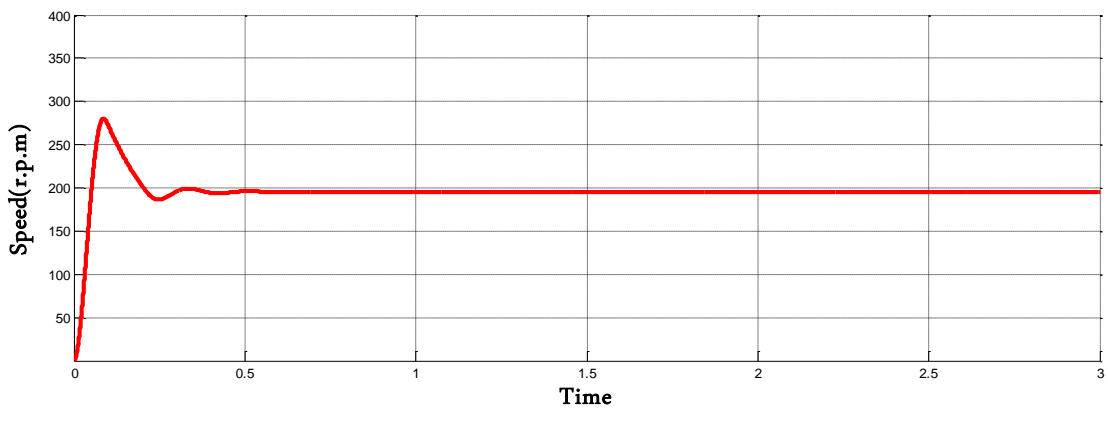

شكل(5) استجابة سرعة المحرك الثاني عند (م=50\%)

2

هناك تقنيات عديدة للسيطرة في هذا المجال وقد تم تناول اثتتان منها في البحث الحالي:

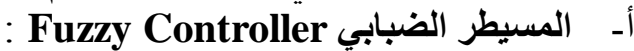

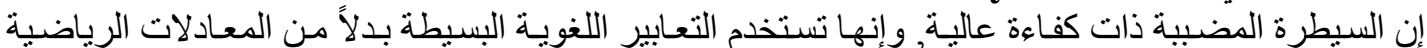

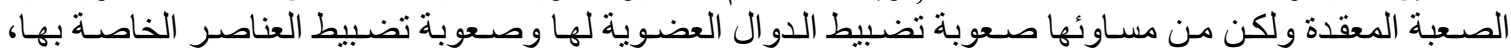

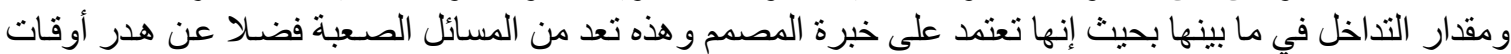

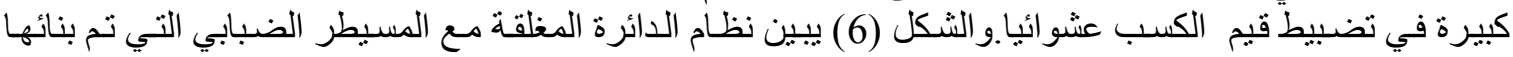

الثكل (7) يمثل استجابة النموذج الاول لمحرك التيار المستمر وعند سرعة مرجعية (500 r.p.m) حيث نم تنغيم كل

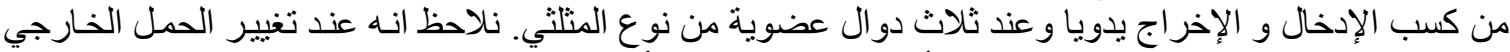

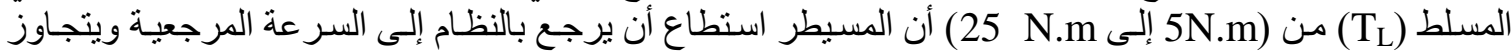

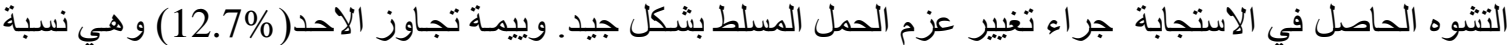



No. 2
April 2013
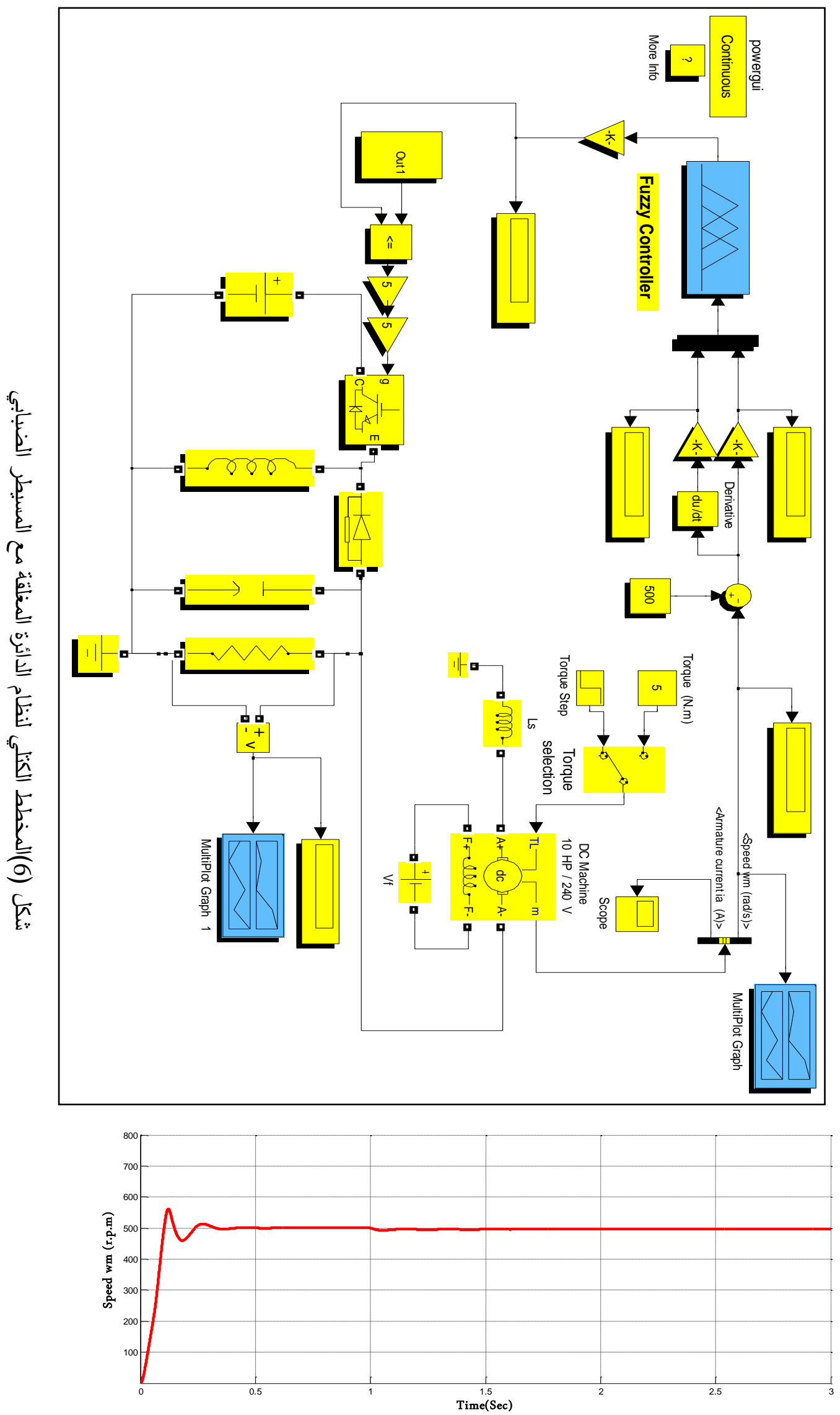

الشكل (7) استجابة السر عة باستخدام المسيطر الضبابي و عند سر عة مرجعية (500 r.p.m) 


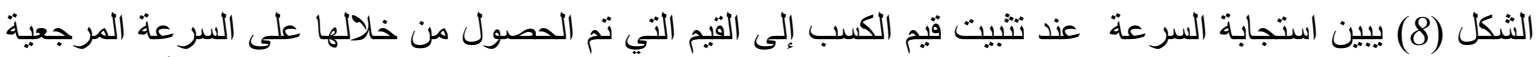

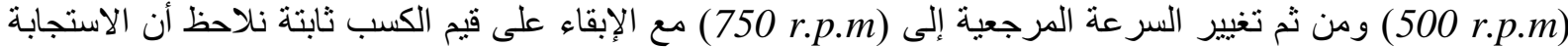

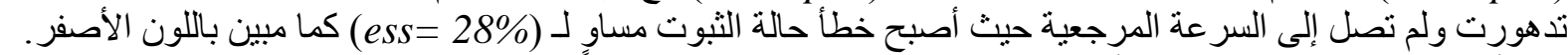

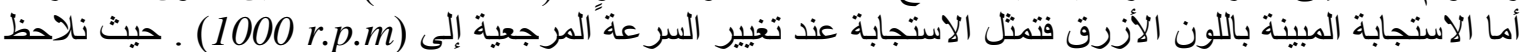

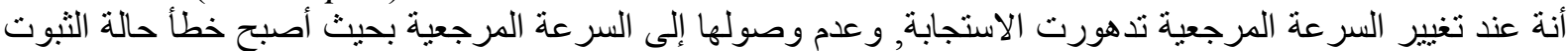

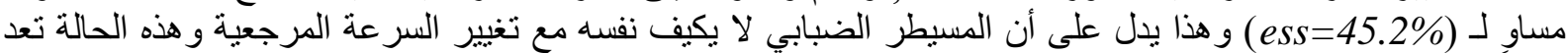

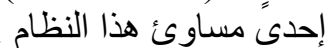

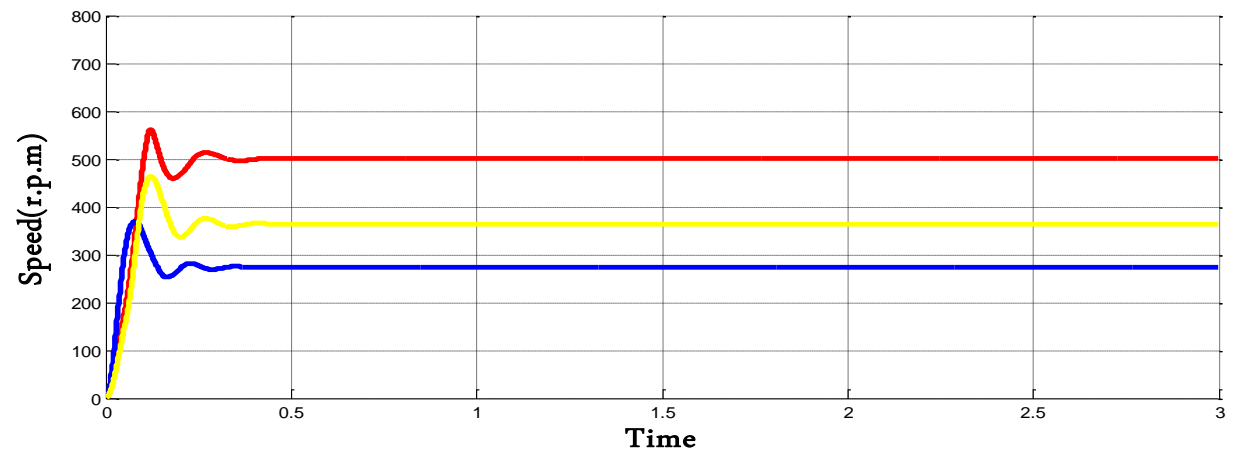

الثكل (8) استجابة سرعة المحرك عند سرع مرجعية مختلفة

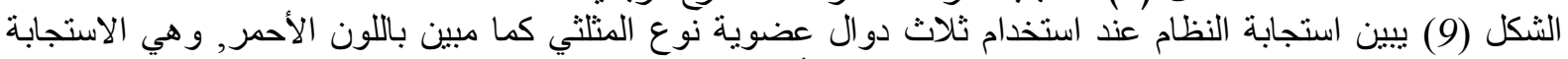

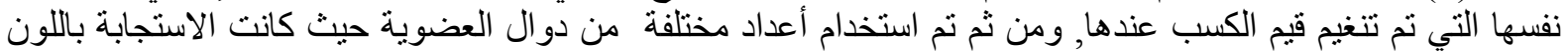

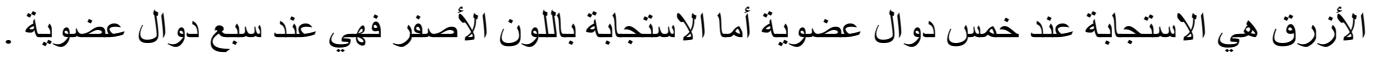

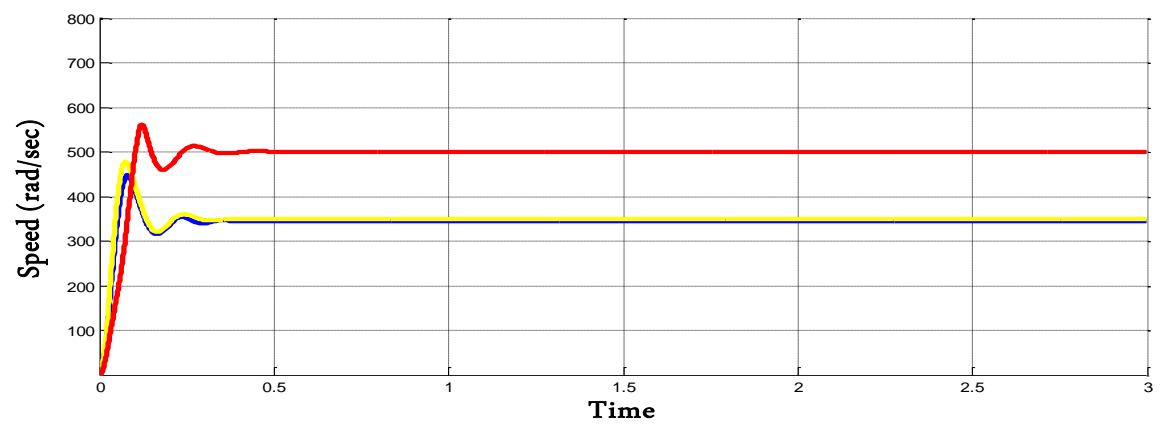

الثكل (9) استجابة سرعة المحرك عندد اعداد مختلفة من دوال العضوية العزية

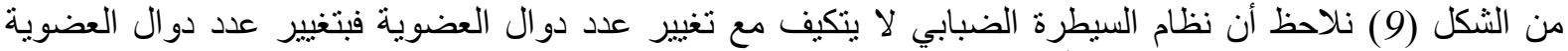

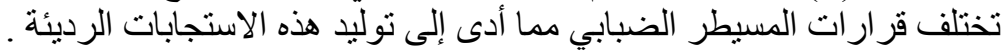

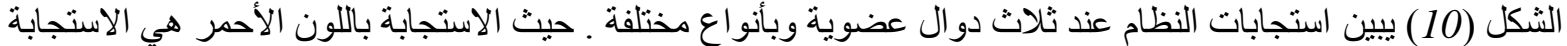

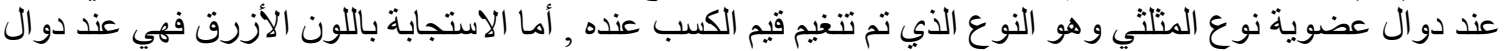

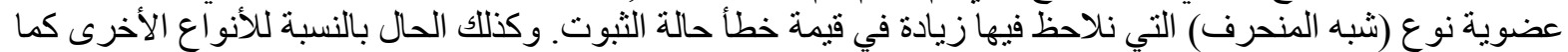

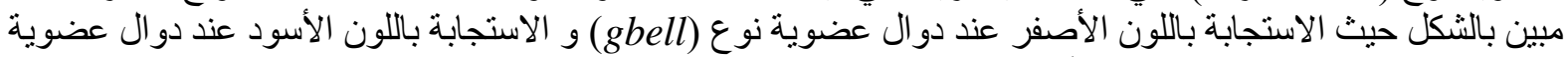

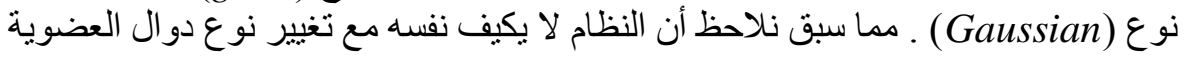

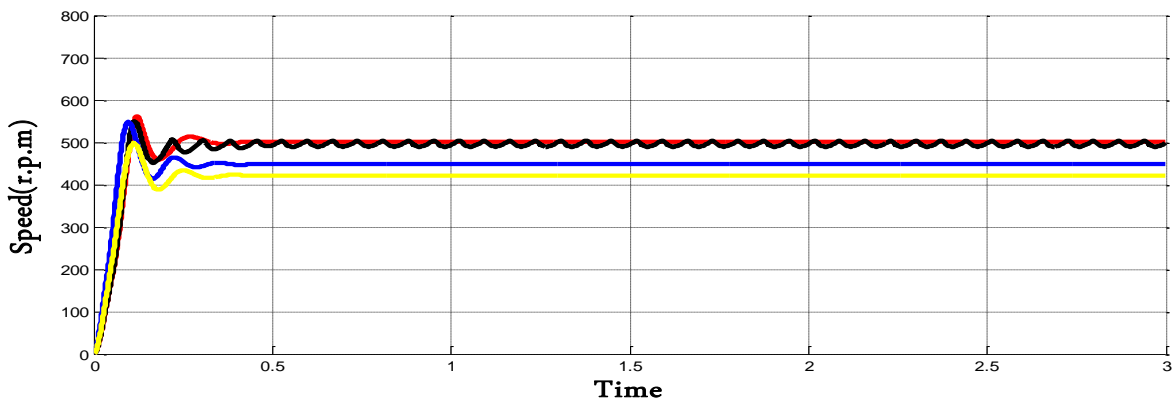

شكل (10) استجابة سر عة المحرك عند أنو اع مختلفة من دوال العضوية 


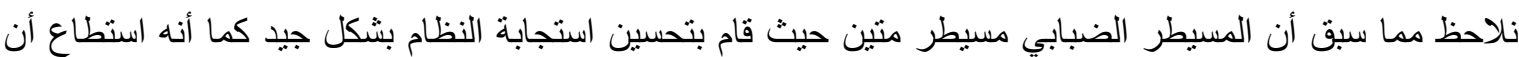

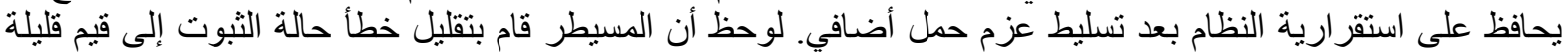

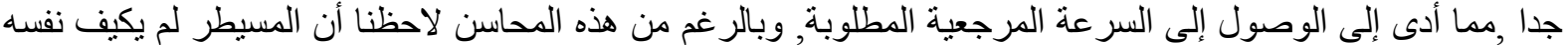
مع تغيير السرعة المرجعية أو تغيير نوع أو الو عدد دوال العضوية .

\section{ب- مسيطر الثبكات العصبية المضببة:}

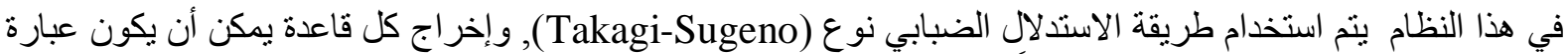

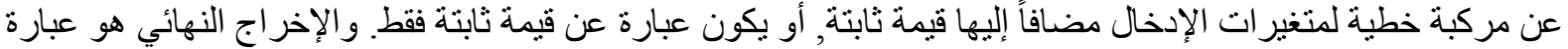

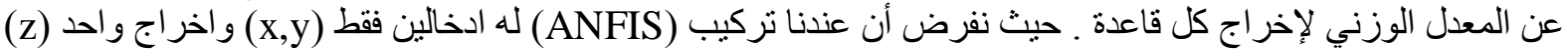

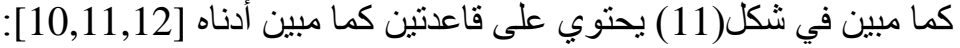

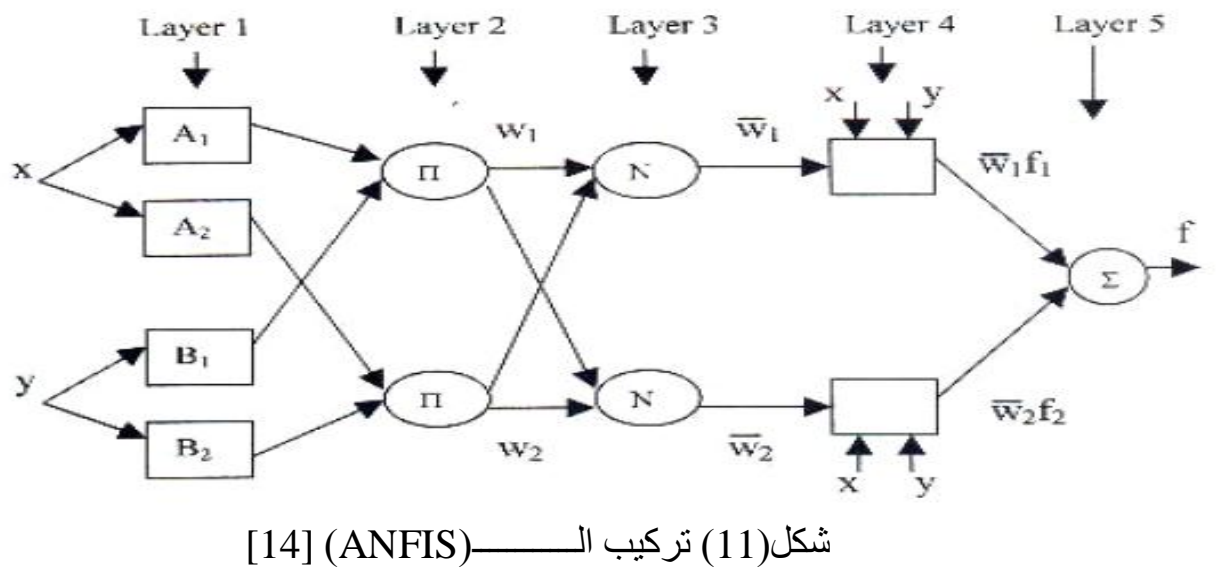

يتكون نظام سبطرة الثبكات العصبية المضيبة الذي نم تصميمه من جز أين , جزء برمجي و هو الجزء الذي تتم فيه عملية

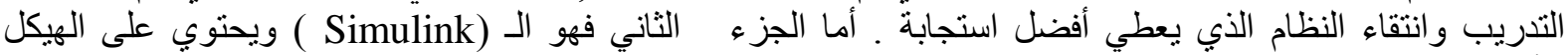

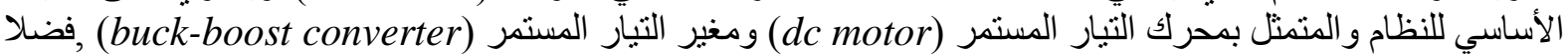

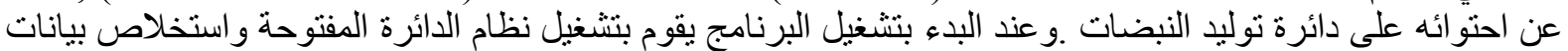

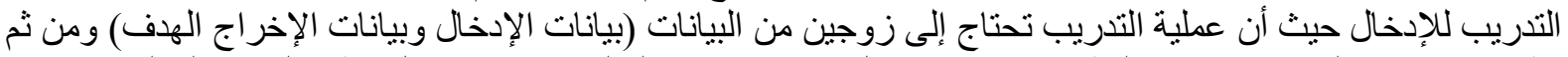

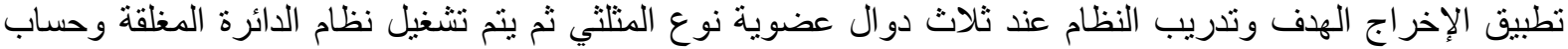

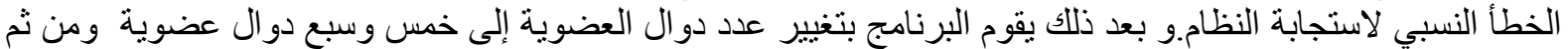

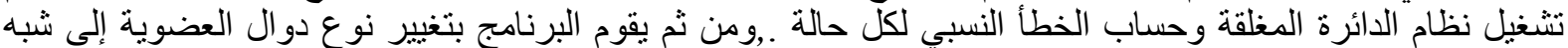

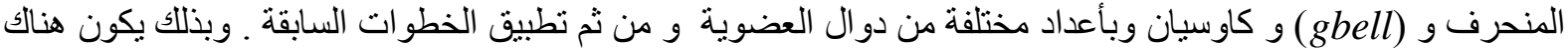

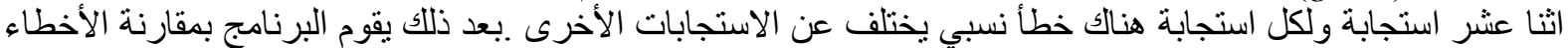

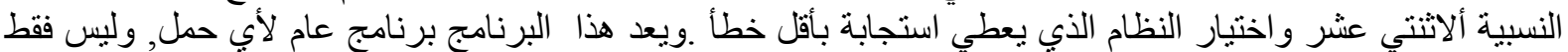

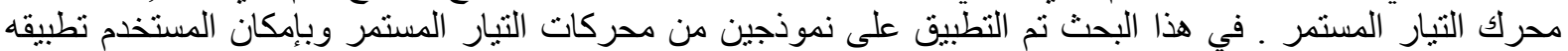

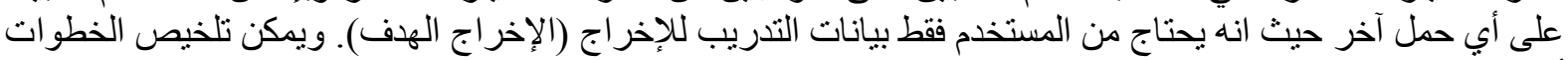
أعلاه بألمخطط الانسيابي المبين في شكل آنس (12). 


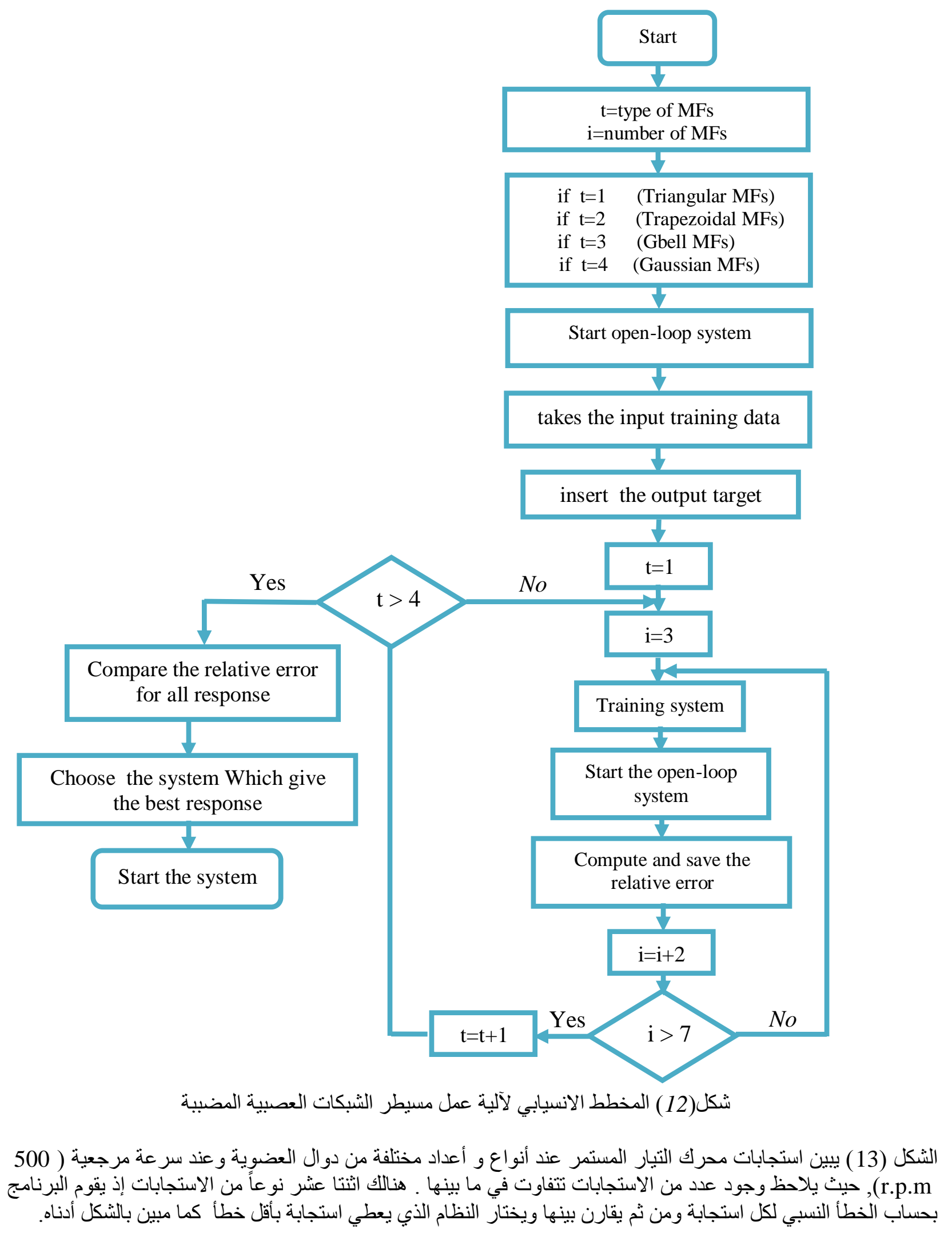




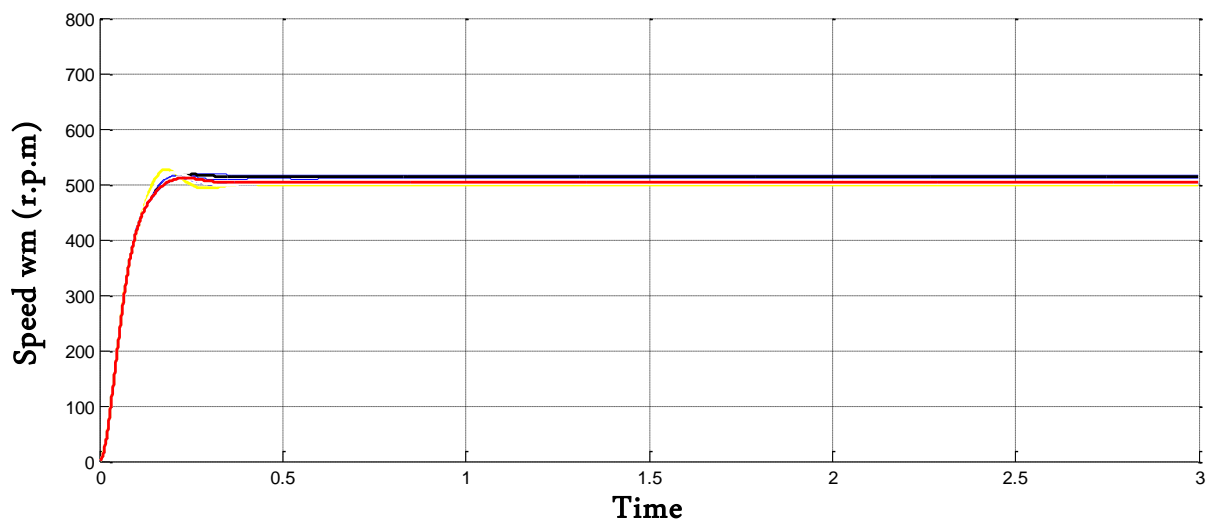

شكل (13) استجابات السرعة مع المسيطر (ANFIS) عند السرعة المرجعية ( 500 r.p.m)

الجدول (1) بيين عملية المقارنة, يلحظ من الجدول أن النظام الذي له ثلاث دوال عضوية نوع شبه المنحرف

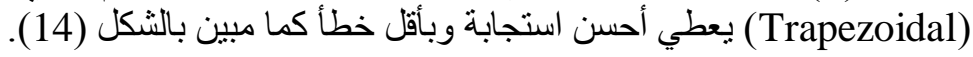

جدول (1) الأخطاء النسبية لاستجابات المحرك عند السرعة (500 r.p.m)

\begin{tabular}{|c|c|c|c|c|}
\hline \multirow{2}{*}{\multicolumn{2}{|c|}{$\mathrm{W}_{\text {ref }}=500$ (r.p.m) }} & \multicolumn{3}{|c|}{ Number of membership function } \\
\hline & & 3 & 5 & 7 \\
\hline \multirow{4}{*}{ 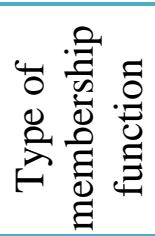 } & Triangular & 0.0778 & 0.0833 & 2.312 \\
\hline & Trapezoidal & 0.07206 & 2.602 & 2.703 \\
\hline & Gbell & 0.0788 & 2.515 & 0.0991 \\
\hline & Gaussian & 0.0728 & 0.08570 & 0.5289 \\
\hline
\end{tabular}

نلاحظ إن قيمة تجاوز الحد للاستجابة التي تم اختبار ها تساوي (Mo.sh=4\%) و (ess=0.0034) وهي نسبة جيدة إذا ما

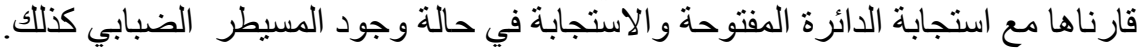

شكل (14) يبين الاستجابة التي تم اختيار ها بواسطة البرنامج التي تحتوي على اقل خطأ نسبي حيث من الجدول نلاحظ

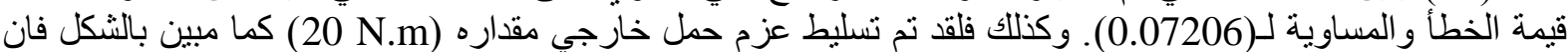
النظام قد تغلب على التشوه الناجم من العزم الإضافي المسلط.

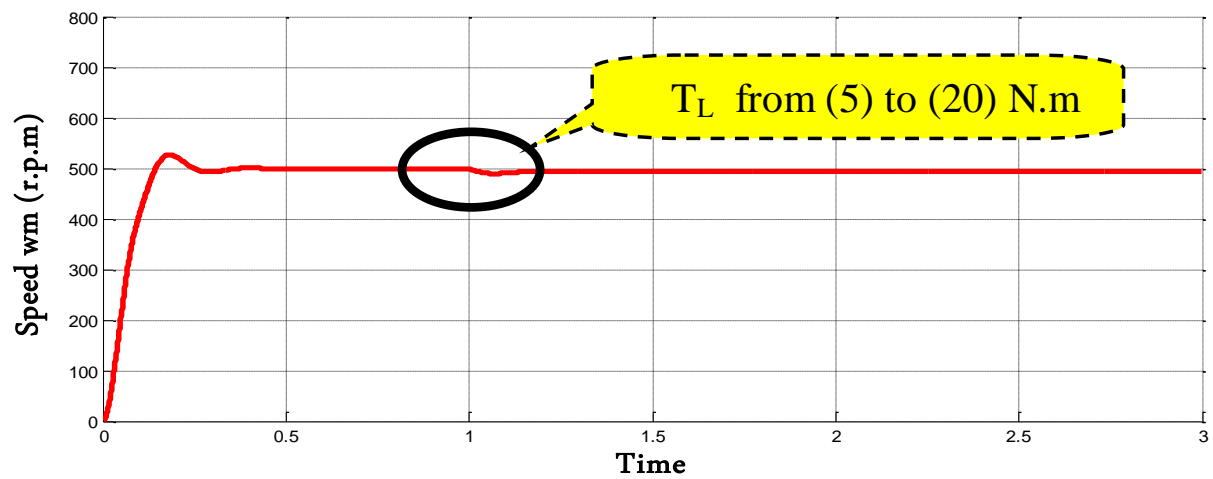

(500 r.p.m) شكل (14) الاستجابة المختارة عند سرعة مرجعية

الثكل (15) يبين استجابات محرك التبار المستمر و عند سر عة مرجعية (750 r.p.m), و الجدول (2) يبين عملية المقارنة فمن الجدول نلاحظ أن النظام الذي له ثلاث دو ال عضوية نوع (Gbell) يعطي أحسن استجابة وبأقل خطأ وكما مبين بالثكل (16). 


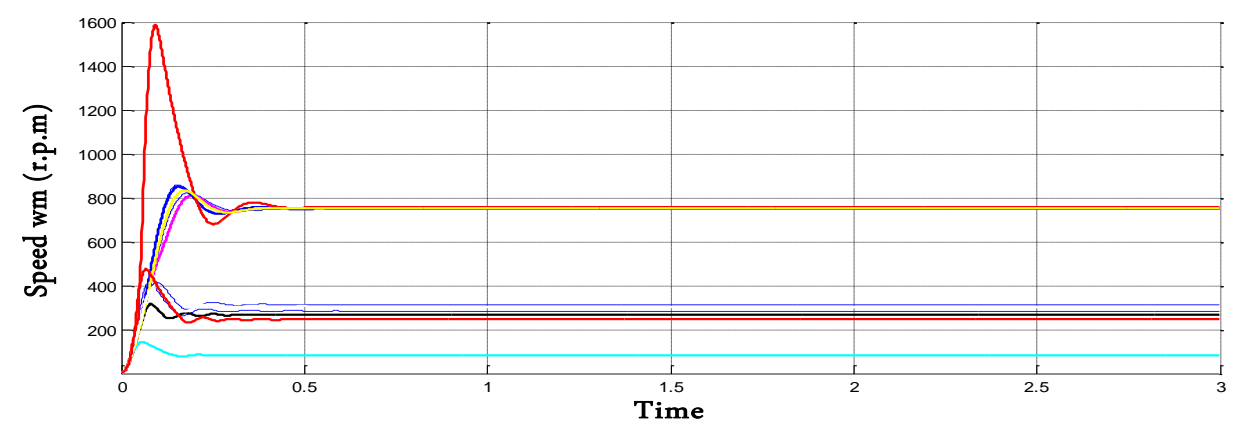

شكل (15) استجابات السر عة للمحرك و عند سر عة مرجعية ( 750 r.p.m )

جدول (2) الأخطاء النسبية لاستجابات المحرك عند سرعة مرجعية (750 r.p.m)

\begin{tabular}{|c|c|c|c|c|}
\hline \multirow{2}{*}{\multicolumn{2}{|c|}{$\mathrm{W}_{\text {ref }}=750$ (r.p.m) }} & \multicolumn{3}{|c|}{ Number of membership function } \\
\hline & & 3 & 5 & 7 \\
\hline \multirow{4}{*}{ 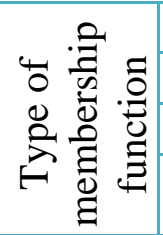 } & Triangular & 0.0855 & 0.00887 & 0.1864 \\
\hline & Trapezoidal & 0.00835 & 0.1944 & 0.2667 \\
\hline & Gbell & 0.00817 & 0.1756 & 0.0171 \\
\hline & Gaussian & 0.00861 & 0.1791 & 0.2007 \\
\hline
\end{tabular}

شكل(16) يبين الاستجابة التي نم اختبار ها بواسطة البرنامج والتي تحتوي على اقل خطأ نسبي فمن الجدول نلاحظ قيمة

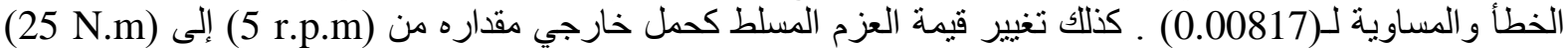
كما مبين بالثكل إذ تغلب النظام على التشوه الناجم من العزم الإضافي المسلط .

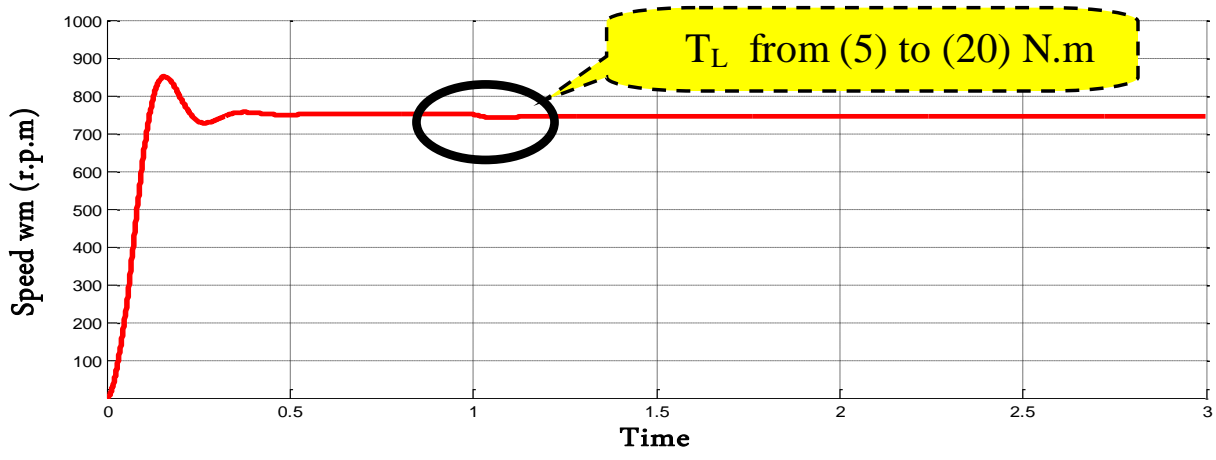

(750 r.p.m) شكل (16) الاستجابة المختارة عند سر عة مرجعية

شكل (17) يبين استجابات محرك التبار المستمر عند سرعة مرجعية (1000 r.p.m) , وفي هذه الحالة تم اختيار النظام

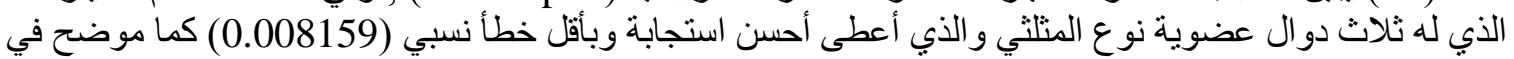
جدول (3) وشكل (18).

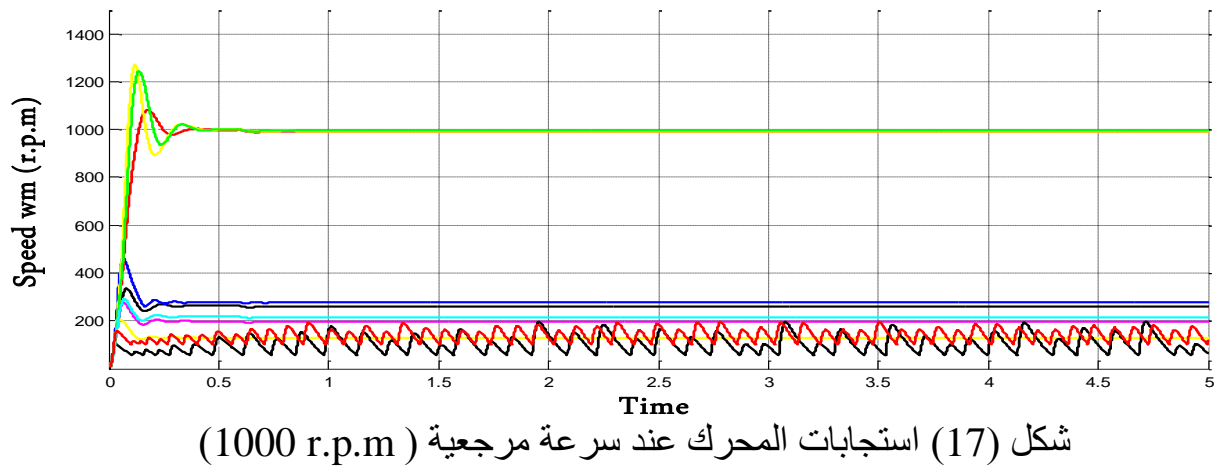




$$
\text { جدول (3) الأخطاء النسبية لاستجابات المحرك عند سر عة مرجعية (1000 r.p.m) }
$$

\begin{tabular}{|c|c|c|c|c|}
\hline \multirow{2}{*}{\multicolumn{2}{|c|}{$\mathrm{W}_{\text {ref }}=1000$ (r.p.m) }} & \multicolumn{3}{|c|}{ Number of membership function } \\
\hline & & 3 & 5 & 7 \\
\hline \multirow{4}{*}{ 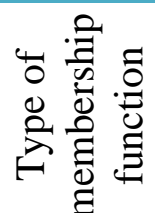 } & Triangular & 0.08159 & 0.74379 & 0.7203814 \\
\hline & Trapezoidal & 0.0798165 & 0.902507 & 0.080681 \\
\hline & Gbell & 0.8038 & 0.917884 & 0.084752 \\
\hline & Gaussian & 0.78611 & 0.87723 & 0.894 \\
\hline
\end{tabular}

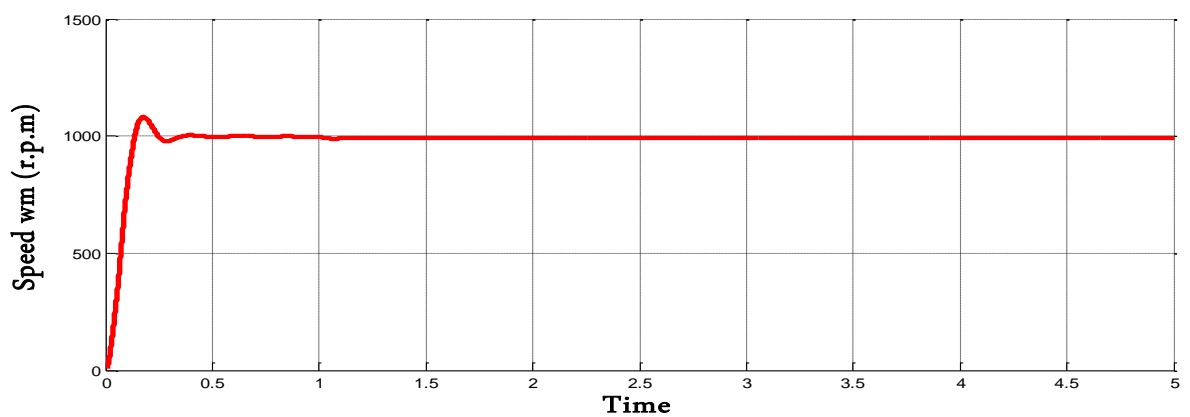

شكل(18) الاستجابة المختارة عند سرعة مرجعية (1000 r.p.m)

كما ذكر سابقا فان مسيطر الثبكات العصبية المضبية المصمم هو مسيطر عام يصلح لأي حمل و لإثبات ذللك تم تغيير نموذج محرك التيار المستمر والموجود ضمن بيئة (Matlab/Simulink). والجدول

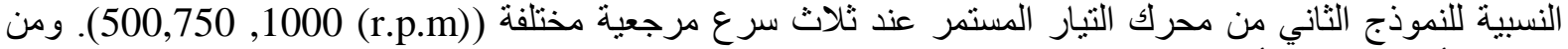

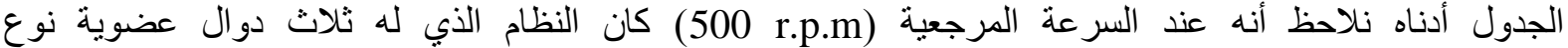
(trapezoidal)

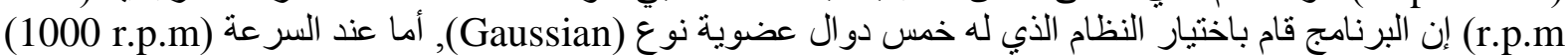

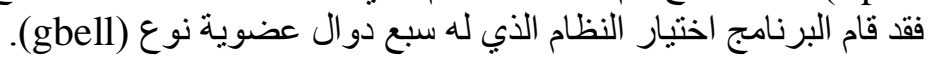

$$
\text { جدول (4) الأخطاء النسبية لاستجابات النموذج الثاني لمحرك التيار المستمر عند السرع المرجعية }
$$

\begin{tabular}{|c|c|c|c|c|c|}
\hline \multirow{2}{*}{\multicolumn{3}{|c|}{ No .of Membership Function }} & \multicolumn{3}{|c|}{ Relative Error } \\
\hline & & & 3 & 5 & 7 \\
\hline \multirow{4}{*}{ 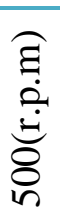 } & \multirow{4}{*}{ 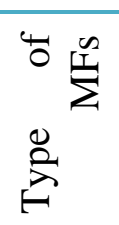 } & Triangular & 0.122 & 0.1588 & 0.1463 \\
\hline & & Trapezoidal & 0.0732 & 0.0754 & 0.07645 \\
\hline & & Gbell & 0.865 & 0.0792 & 0.0772 \\
\hline & & Gaussian & 0.0788 & 0.0891 & 0.0895 \\
\hline \multirow{4}{*}{ 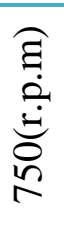 } & \multirow{4}{*}{ 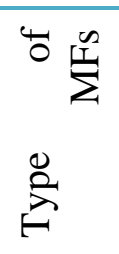 } & Triangular & 0.08159 & 0.74379 & 0.7203814 \\
\hline & & Trapezoidal & 0.0798165 & 0.902507 & 0.080681 \\
\hline & & Gbell & 0.8038 & 0.917884 & 0.084752 \\
\hline & & Gaussian & 0.78611 & 0.87723 & 0.894 \\
\hline \multirow{4}{*}{ 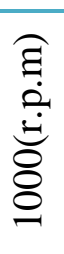 } & \multirow{4}{*}{ 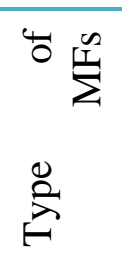 } & Triangular & 0.08159 & 0.74379 & 0.7203814 \\
\hline & & Trapezoidal & 0.0798165 & 0.902507 & 0.080681 \\
\hline & & Gbell & 0.8038 & 0.917884 & 0.084752 \\
\hline & & Gaussian & 0.78611 & 0.87723 & 0.894 \\
\hline
\end{tabular}
(500 , 750 ,1000 r.p.m) 


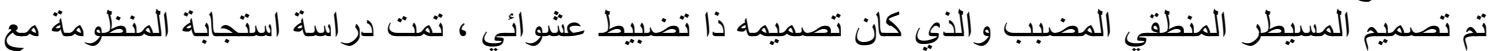

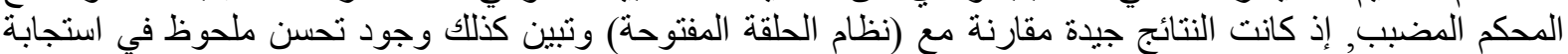

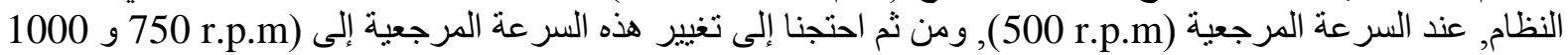

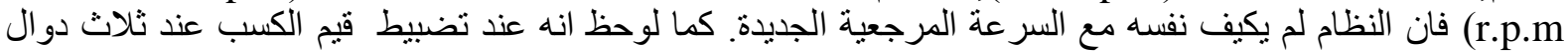
العضوية نوع ألمثلثي ومن ثم تغيير نوع أو عدد دوال العضوية بحيث أصبحت الاستجابة رديئة وان المبنة المسيطر لم يكيف

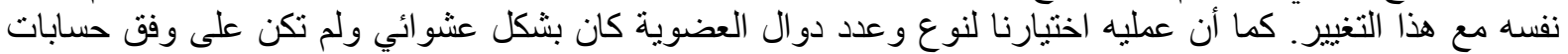

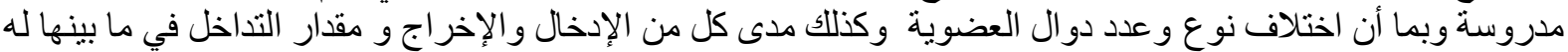
تأثثير على استجابة النظام, ولذلك لابد أن أنكون هناك طريقة مدروسة وقاعدة يستند عليها النظام في عملية الاختيار.

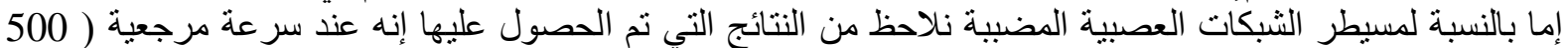

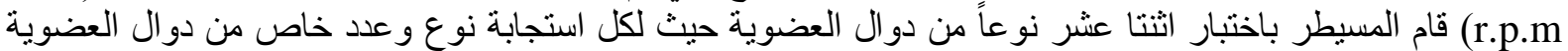

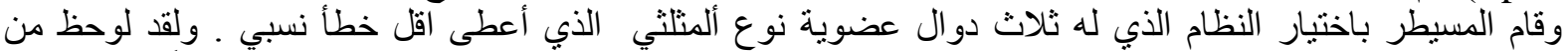

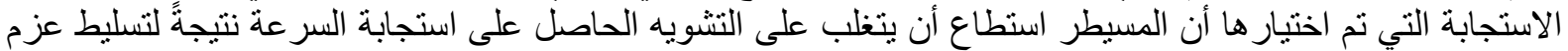

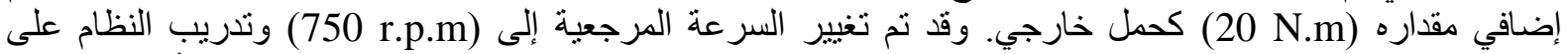

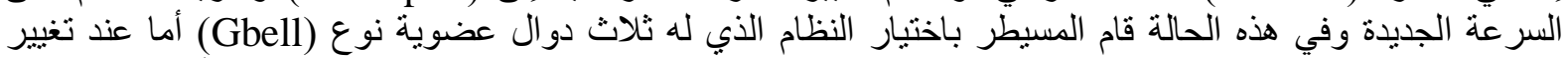

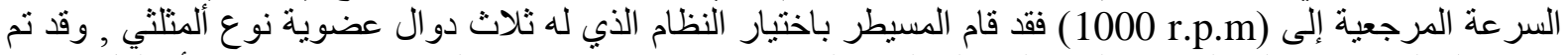

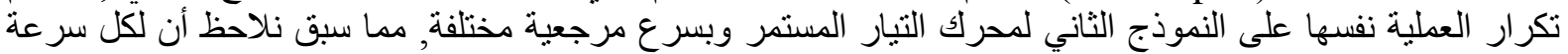
مرجعية وجود نوع و عدد خاص من دو آل العضوية يعطي أفضل استجابة التئة

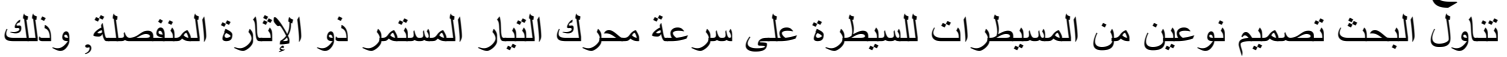
الاستنتاج

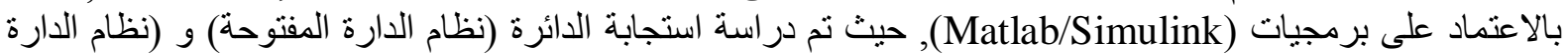

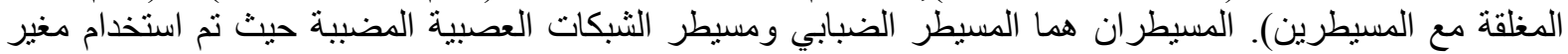

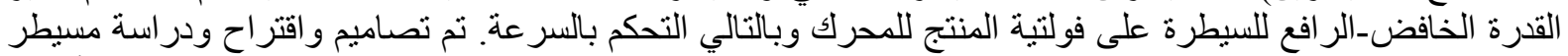

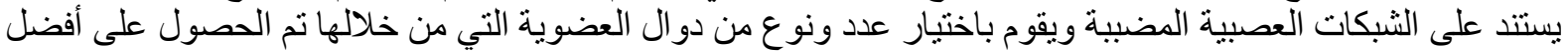

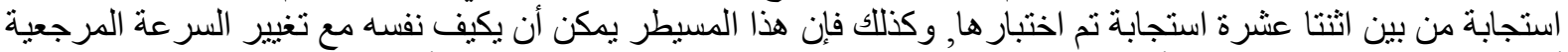

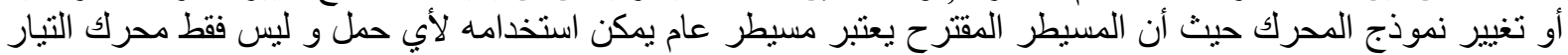

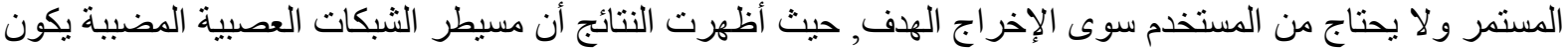
ذا كفاءة أعلى ويعطي نتائج أفضل إذا ما قورن بالمسيطر الضبابي الإفي

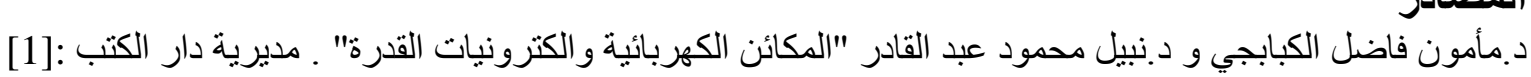
للطباعة و النشر رجامعة الموصل.

[2]: Jun Oh JANG and Pyeong Gi LEE, 'Neuro-Fuzzy Control for DC Motor Friction Compensation" Department of computer control Engineering ,south korea.2000.

[3]: Adriana Florescu, Ovidiu Grigore, Dan Alexandru Stoichescu, Alexandru Vasile, "NEURO- FUZZY DESIGN IN DC MOTOR SPEED CONTROL" University of Bucharest, Electronics and Telecommunications Faculty, Bd. Armata Poporului 1-3, Sect. 6, 77206 - Bucharest, Romania .2001.

[4]: Ferenc Farkas, Sandor Halasz, Istvan Kadar, 'Speed Sensorless Neuro-Fuzzy Controller for Brush type DC Machine', Department of Electric power Engineering ,Budpest University of Technology ,Hungary2005.

[5]: Salwan Samir Sabri Ali, "Optimal Fuzzy Controller Design For (Cuk) Converter Circuit Using Genetic Algorithm", thesis M.Sc. University of Mosul/ Iraq,2008. 
[6]: Mohamed H. Rashid, "Power Electronics Circuits , Devices and Applications", Third Edition ,Electrical and Engineering, University of West Florida,2004.

[7]- Stanimir Yordanov Yordanov, "An Approach on T-S Fuzzy Model and Control of Buckboost Converter ",Department AIUT, TU Gabrovo, H.Dimitar 4., 5300, Bulgaria,.2004........buck-boost

[8]: Nadia Qassim Mohammed, "Neuro Fuzzy Controller For A Four Quadrant Position and Speed Control", University of Baghdad/ Iraq, , thesis,M.Sc.2005.

[9]: Maher Mohammed Fawzi Saber AL Greer, "Design and Implementation Of Computerized Industrial Fuzzy Control System “,University of Mosul/ Iraq, , thesis ,M.Sc. 2004.

[10]: Leond Reznik, "Fuzzy Controller", Vectoria University of Technology ,Melbourne, Australia. 1997

[11]- Yodyium Tipsuwan and Saksiri Aiemchareon, "A Neuro-Fuzzy Network-Based Controller for DC Motor Speed Control “,Department of Computer Engineering, Kasetsart University, Bangkok, Thailand.2005 ANFIS

[12]: Nada Abd Al-Ghani, "Speaker Recognition Using Neuro-Fuzzy Method”, thesis ,M.Sc. University of Mosul/ Iraq,2004.

[13]: Mehamet Acar \& Ismail Temiz, "Motion Controller Design For The Speed Control Of DC Servo Motor", Gaziosmanpasa University \& Marmara University , International Journal of Applied Mathematics and Information . 2007.

[14]: Heikki Koivo, “Adaptive Neuro-Fuzzy Inference System”, Paper ,IEEE . 2000.

$$
\text { تم اجراء البحث في كلية ألهندة = جامعة ألموصل }
$$

